\title{
Online Adaptive Error Compensation SVM-Based Sliding Mode Control of an Unmanned Aerial Vehicle
}

\author{
Kaijia Xue, Congqing Wang, Zhiyu Li, and Hanxin Chen \\ College of Automation, Nanjing University of Aeronautics and Astronautics, Nanjing 210016, China \\ Correspondence should be addressed to Kaijia Xue; 464120586@qq.com
}

Received 14 October 2015; Accepted 27 January 2016

Academic Editor: Kenneth M. Sobel

Copyright (C) 2016 Kaijia Xue et al. This is an open access article distributed under the Creative Commons Attribution License, which permits unrestricted use, distribution, and reproduction in any medium, provided the original work is properly cited.

Unmanned Aerial Vehicle (UAV) is a nonlinear dynamic system with uncertainties and noises. Therefore, an appropriate control system has an obligation to ensure the stabilization and navigation of UAV. This paper mainly discusses the control problem of quad-rotor UAV system, which is influenced by unknown parameters and noises. Besides, a sliding mode control based on online adaptive error compensation support vector machine (SVM) is proposed for stabilizing quad-rotor UAV system. Sliding mode controller is established through analyzing quad-rotor dynamics model in which the unknown parameters are computed by offline SVM. During this process, the online adaptive error compensation SVM method is applied in this paper. As modeling errors and noises both exist in the process of flight, the offline SVM one-time mode cannot predict the uncertainties and noises accurately. The control law is adjusted in real-time by introducing new training sample data to online adaptive SVM in the control process, so that the stability and robustness of flight are ensured. It can be demonstrated through the simulation experiments that the UAV that joined online adaptive SVM can track the changing path faster according to its dynamic model. Consequently, the proposed method that is proved has the better control effect in the UAV system.

\section{Introduction}

Unmanned Aerial Vehicle (UAV) has drawn a great attention from researchers in the past few years. The characteristics of quad-rotor UAV, such as novel appearance, simple structure, low cost, excellent performance, and a unique way of flight control $[1,2]$, have enabled it to be the new research hotspot all over the world [3-6].

Quad-rotor UAV consists of four independent propellers attached at each corner of a cross frame. It is relied on the resultant forces and moments of four independent rotors that adjust the flight attitude and position [7]. Compared with the fixed wing aircraft, the quad-rotor UAVs that are equipped with vertical take-off have better landing capabilities even in the narrow space, because this vertical take-off has higher manoeuvrability and hovering capacities [8].

Quad-rotor UAV has all the advantages of unmanned rotor aerial vehicle; thus its application prospect is limitless. UAV has been used more and more in civilian such as rescue missions [9], forest fire surveillance [10], disaster relief support [11], exploration of high buildings or contaminated areas [12], aerial photography [13], area mapping [14], inspection of transmission lines and bridge [15], and assessment of water status [16].

However, the 6-DOF air-frame dynamic of quad-rotor UAV is nonlinear and coupled with each other. In addition, an underactuated system for the aircraft is equipped with four actuators, whose number is smaller than the degrees of freedom. Therefore, it is comparatively difficult to design an excellent controller for aircraft due to the reasons mentioned above.

The conventional sliding mode control (SMC), as a kind of nonlinear system control method, possesses the advantages of wide applicability, fine dynamic characteristics, and insensitivity to interference changes. This research on the sliding model control mainly deals with adaptive sliding model control [17, 18], robust sliding mode control [19], optimal sliding mode control [20], fuzzy slidingmode control 


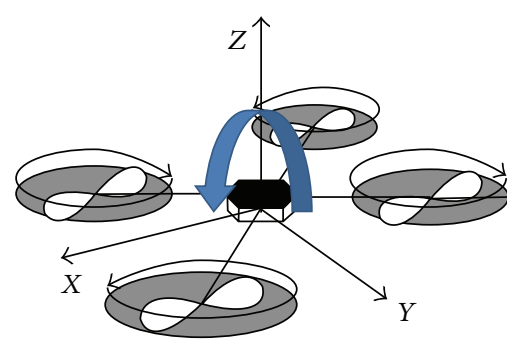

(a) Pitch

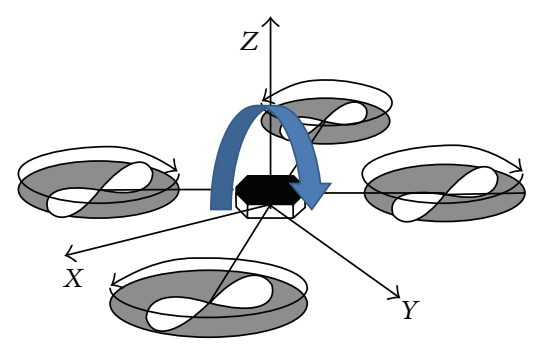

(b) Roll

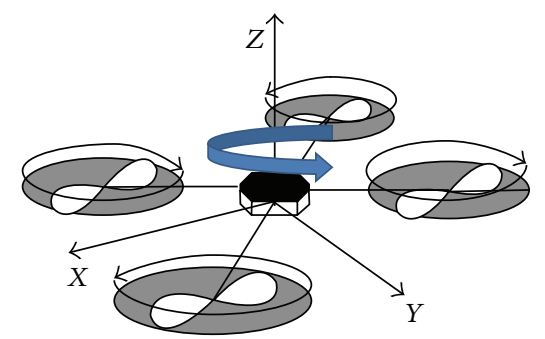

(c) Yaw

FIGURE 1: Three basic movements of quad-rotor UAV.

[21, 22], neural network sliding mode control [23, 24], and sliding mode control combined with other intelligent controls [25].

To achieve the effective sliding mode control of quadrotor UAV, the mathematical model of various flight conditions must be designed accurately. Nevertheless, during the process of flight, UAVs are affected by various physical attributes such as aerodynamic force, gravity, gyroscopic effect, and the rotor inertia moment. It is also very vulnerable to the interference of the external environment like airflow. According to these reasons, it is quite difficult to establish a reliable dynamic model. Furthermore, the size and the weight of the rotor, the degree of deformation, and parameters of aerodynamic performance will also directly affect the precision of the model.

In consideration of these problems, how to make the estimator more accurate is very important. Hence the system can reach the sliding surface rapidly in coordination with a sliding mode control law.

Standard offline support vector machine (SVM) is a machine learning based on statistical theory. It can solve small-sample, nonlinear, and high dimension problems by using structural risk minimization. This method can efficiently supply the details without considering the changes of modeling the quad-rotor system. But it only focuses on training sample data within the limited time and its estimated result is predicted one time; hence there are still many dynamic variations that cannot be predicted during the process of flight. The main contribution of this paper is to compensate unknown parameters and to reduce the dynamic error by using online adaptive SVM for the quadrotor system.

This paper presents a method that standard SVM is combined with online adaptive error compensation SVM to design a sliding mode controller. Through this way, the weights of SVM can be adjusted adaptively to an optimal status. The rest of the paper is divided into five sections. The nonlinear dynamic model of the quad-rotor is described in Section 2. The design procedure of sliding mode controller using the online adaptive error compensation SVM is presented in Section 3. Section 4 analyzes the stability of online adaptive error compensation SVM. Simulation results are shown in Section 5. Section 6 draws the concluding remarks. All in all, this paper proves the effectiveness of this method through the simulation results of the quad-rotor system.

\section{Dynamic Model Analysis of Quad-Rotor UAV}

The quad-rotor UAV is an underactuated system as it has six degrees of freedom with only four inputs. Pitch movement of quad-rotor UAV is motivated by reducing two motors' speed on either front or back side. And an equivalent increase of the two motors' speed on another side is required simultaneously. According to the symmetrical structure of quad-rotor UAV, the adjustment mode of the roll movement is similar to the pitch movement.

Yaw movement of quad-rotor UAV is realized through reducing one diagonal motors' speed, and equally increasing the two motors' speed of another diagonal at the same time. Based on this theory, the reduced speed on motor torque of the quad-rotor UAV is less than the speed increased; therefore, it makes the aircraft body present in a framework of yaw movement. Three basic movements of quad-rotor are shown in Figure 1.

Two coordinate systems are defined before the dynamic model is established, one for the ground coordinate system and the other for quad-rotor coordinate system. The ground coordinate system is a static coordinate system that relates to a reference ground point. The flight speed and displacement of quad-rotor UAV in different directions are mainly judged by aiming at the ground coordinate system. Pitch angle, roll angle, and yaw angle are mainly considered from the direction of the quad-rotor coordinate system which is based on the geometry center of the aircraft body. The speed of the aircraft under the body coordinate system is described as the following relationship:

$$
V=\left[\begin{array}{c}
u \\
v \\
w
\end{array}\right]=\left[\begin{array}{lll}
R_{1} & R_{2} & R_{3}
\end{array}\right]\left[\begin{array}{c}
v_{q x} \\
v_{q y} \\
v_{q z}
\end{array}\right]=\left[\begin{array}{lll}
R_{1} & R_{2} & R_{3}
\end{array}\right]\left[\begin{array}{c}
\dot{x} \\
\dot{y} \\
\dot{z}
\end{array}\right] .
$$

As shown in Figure 2, $V=[u, v, w]^{T}$ is the velocity vector of the plane with respect to the ground coordinate system and $\left[v_{q x}, v_{q y}, v_{q z}\right]^{T}$ is the velocity vector with respect to the quadrotor coordinate system. The transformation matrix $R=$ $\left[R_{1}, R_{2}, R_{3}\right]^{T}$ can be denoted as follows: 


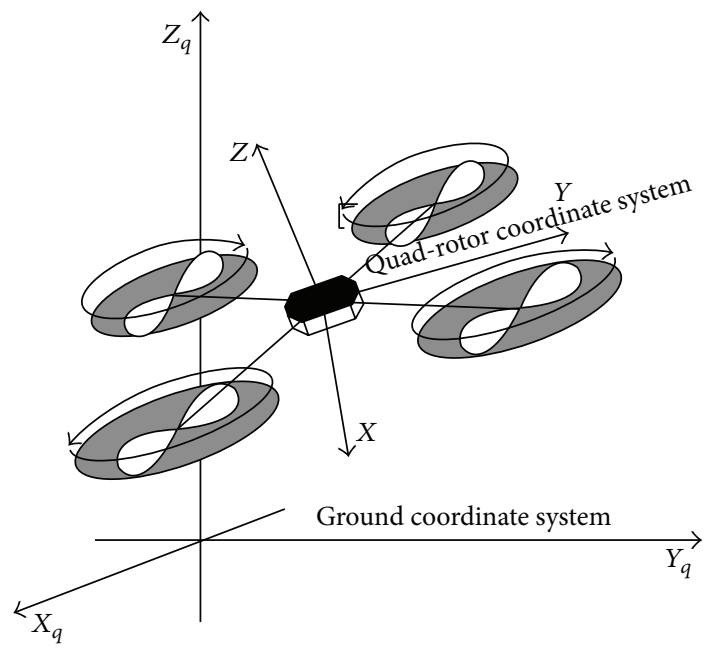

FIGURE 2: Relationship between the quad-rotor coordinate system and the ground coordinate system.

$$
R=\left[\begin{array}{ccc}
\cos \phi \cos \theta & -\sin \phi \cos \varphi+\cos \phi \sin \theta \sin \varphi & \sin \varphi \sin \phi+\cos \phi \sin \theta \cos \varphi \\
\sin \phi \cos \theta & \cos \phi \cos \varphi+\sin \phi \sin \theta \sin \varphi & -\cos \phi \sin \varphi+\sin \phi \sin \theta \cos \varphi \\
-\sin \theta & \cos \theta \sin \varphi & \cos \theta \cos \varphi
\end{array}\right]
$$

where $\phi$ is the pitch angle, $\theta$ is the roll angle, and $\varphi$ is the yaw angle. According to Newton's second law, the quad-rotor motion equation of ground coordinate system can be written as

$$
\frac{d(m V)}{d t}=\left(F_{G}+F_{T}+F_{R}\right)=F,
$$

where the response of quad-rotor UAV produced by gravity is

$$
F_{G}=m g G_{1}
$$

where $m$ is the mass of UAV and $g$ is the acceleration of gravity. Each propeller generates vertical lifting force, so the overall lifting force $F_{T}$ will be

$$
F_{T}=k_{b} \sum_{i=1}^{4} \Omega_{i}^{2} G_{2}=T G_{2},
$$

where the $k_{b}$ is lifting force coefficient, $T=k_{b}\left(\Omega_{1}^{2}+\Omega_{2}^{2}+\right.$ $\left.\Omega_{3}^{2}+\Omega_{4}^{2}\right)$, and $\Omega_{i}$ means the rotation rate of the $i$ th propeller. $G_{1}$ and $G_{2}$ are transfer matrices. Air resistance of quad-rotor $\mathrm{UAV}$ is expressed as

$$
F_{R}=\left[-k_{R 1} u,-k_{R 2} v,-k_{R 3} w\right]^{T} \text {. }
$$

Let $G_{1}=G_{2}=[0,0,1]^{T}$; by taking (3), (4), and (5) into (6), then the motion equations of quad-rotor UAV can be written as

$$
\left[\begin{array}{c}
\dot{u} \\
\dot{v} \\
\dot{w}
\end{array}\right]
$$

$$
=\left[\begin{array}{c}
-\frac{T}{m}(\sin \phi \sin \varphi+\cos \phi \cos \theta \cos \varphi)-k_{R 1} \dot{x} \\
-\frac{T}{m}(-\cos \phi \sin \varphi+\sin \phi \sin \theta \cos \varphi)-k_{R 2} \dot{y} \\
g-\frac{T}{m}(\cos \varphi \cos \theta)-k_{R 3} \dot{z}
\end{array}\right] .
$$

Attitude foundation model can cooperate with the two coordinate systems from (7) by establishing the aircraft angular velocity. On the basis of conversion relations between the aircraft coordinate system and the ground coordinate system, the following quad-rotor attitude kinematics equation can be got:

$$
\left[\begin{array}{c}
\dot{\phi} \\
\dot{\theta} \\
\dot{\psi}
\end{array}\right]=\left[\begin{array}{ccc}
1 & \sin \phi \tan \theta & \cos \phi \tan \theta \\
0 & \cos \phi & -\sin \phi \\
0 & \frac{\sin \phi}{\cos \theta} & \frac{\cos \phi}{\cos \theta}
\end{array}\right]\left[\begin{array}{l}
p \\
q \\
r
\end{array}\right] .
$$


According to the moment of momentum theorem, the aircraft torque equation of mass motion can be derived by Newton-Euler equation:

$$
\frac{d(J \omega)}{d t}=\left(M_{R}+M_{G}+M_{D}+M_{d}\right)=M,
$$

where $\omega=[p, q, r]^{T}$ is the rotation rate of quadrotor UAV based on the aircraft coordinate system. $J=$ $\operatorname{diag}\left[J_{x}, J_{y}, J_{z}\right]$ means the moment of inertia of quad-rotor UAV. $M_{R}, M_{G}, M_{D}$, and $M_{d}$ represent rotor lift, gyroscopic effect generated by the rotor, air resistance torque, and external disturbances torque, respectively. $M$ is overall external torque of quad-rotor UAV.

The torque comes from four rotor wings. The relationship between air dynamic torque and four motors' speeds $\Omega_{1}, \Omega_{2}$, $\Omega_{3}$, and $\Omega_{4}$ is

$$
\begin{aligned}
M_{R} & =\left[\begin{array}{c}
\tau_{\phi} \\
\tau_{\theta} \\
\tau_{\psi}
\end{array}\right] \\
& =\left[\begin{array}{cccc}
-\frac{\sqrt{2}}{2} l k_{b} & \frac{\sqrt{2}}{2} l k_{b} & \frac{\sqrt{2}}{2} l k_{b} & -\frac{\sqrt{2}}{2} l k_{b} \\
\frac{\sqrt{2}}{2} l k_{b} & \frac{\sqrt{2}}{2} l k_{b} & -\frac{\sqrt{2}}{2} l k_{b} & -\frac{\sqrt{2}}{2} l k_{b} \\
k_{d} & -k_{d} & k_{d} & -k_{d}
\end{array}\right]\left[\begin{array}{c}
\Omega_{1}^{2} \\
\Omega_{2}^{2} \\
\Omega_{3}^{2} \\
\Omega_{4}^{2}
\end{array}\right],
\end{aligned}
$$

where $\tau_{\phi}, \tau_{\theta}$, and $\tau_{\varphi}$ mean the three-axis torques caused by brush-less motors, $l$ is the distance from the motors to the center of the aircraft, $k_{b}$ is the lift coefficient, and $k_{d}$ is the torque coefficient. The torque of the gyroscopic effect $M_{G}$ is

$$
M_{G}=\left[\begin{array}{c}
\tau_{G \phi} \\
\tau_{G \theta} \\
\tau_{G \psi}
\end{array}\right]=\left[\begin{array}{c}
J_{r x} \Omega^{\prime} q \\
-J_{r y} \Omega^{\prime} p \\
0
\end{array}\right],
$$

where $\Omega^{\prime}=\Omega_{1}-\Omega_{2}+\Omega_{3}-\Omega_{4}$ and $J_{r x}, J_{r y}$ are the rotor moments of inertia that rotate around of the motor shaft. The air resistance torque vector $M_{D}$ is

$$
M_{D}=\left[\begin{array}{c}
\tau_{D \phi} \\
\tau_{D \theta} \\
\tau_{D \psi}
\end{array}\right]=k_{D} \omega=\left[\begin{array}{c}
k_{D x} p \\
k_{D y} q \\
k_{D z} r
\end{array}\right],
$$

where $k_{D}=\operatorname{diag}\left[k_{D x}, k_{D x}, k_{D x}\right]$ and $k_{D x}, k_{D x}$, and $k_{D x}$ are moment coefficients of three-axis air resistance in the aircraft coordinate system. Finally (13) can be obtained by

$$
\left[\begin{array}{c}
\dot{p} \\
\dot{q} \\
\dot{r}
\end{array}\right]=\left[\begin{array}{l}
\frac{J_{y}-J_{z}}{J_{x}} q r+\frac{\tau_{G \phi}}{J_{x}}+\frac{\tau_{\phi}}{J_{x}}+\frac{\tau_{D \phi}}{J_{x}} \\
\frac{J_{z}-J_{x}}{J_{y}} p r+\frac{\tau_{G \theta}}{J_{y}}+\frac{\tau_{\theta}}{J_{y}}+\frac{\tau_{D \theta}}{J_{y}} \\
\frac{J_{x}-J_{y}}{J_{z}} p q+\frac{\tau_{G \psi}}{J_{z}}+\frac{\tau_{\psi}}{J_{z}}+\frac{\tau_{D \psi}}{J_{z}}
\end{array}\right] .
$$

\section{Sliding Mode Controller Design of Unmanned Aerial Vehicle}

In this section, the purpose is to carry out the design procedure of basic sliding mode controller. Firstly, sliding surface $s$ is established according to the attitude error that satisfies the necessary sliding condition $s \dot{s}<0$. Secondly, integrate sliding mode control law with equivalent control part and switch control part will be presented. If the attitude angle variation is small and the speed is slow when the aircraft is hovering, a simplification can be made as $[\dot{\phi}, \dot{\theta}, \dot{\psi}]^{T} \approx$ $[p, q, r]^{T}$. The quad-rotor UAV attitude model with sliding mode controller can be written as

$$
\begin{aligned}
\dot{X} & =X_{1}, \\
\dot{X}_{1} & =\mathbf{f}(X)+\mathbf{g}(X) \mathbf{u}+\Delta \mathbf{f}+\mathbf{d} .
\end{aligned}
$$

In this equation, $X=[x, y, z, \phi, \theta, \psi]^{T}, X_{1}$ is the first derivative of $X$, and $\mathbf{f}(X)$ is

$$
\mathbf{f}(X)=\left[\begin{array}{c}
-k_{R 1} \dot{x} \\
-k_{R 2} \dot{y} \\
g-k_{R 3} \dot{z} \\
\frac{J_{y}-J_{z}}{J_{x}} q r+\frac{J_{r z}}{J_{x}} \Omega^{\prime} q+\frac{k_{D x} p}{J_{x}} \\
\frac{J_{z}-J_{x}}{J_{y}} p r-\frac{J_{r z}}{J_{y}} \Omega^{\prime} p+\frac{k_{D y} q}{J_{x}} \\
\frac{J_{x}-J_{y}}{J_{z}} p q+\frac{k_{D z} r}{J_{x}}
\end{array}\right] .
$$

$\mathbf{g}(X)$ is the input matrix, $\Delta \mathbf{f}+\mathbf{d}=\left[d_{x}, d_{y}, d_{z}, d_{\phi}, d_{\theta}, d_{\psi}\right]^{T}$, $\Delta \mathbf{f} \in R^{6}$ is an uncertainty vector, and $\mathbf{d} \in R^{6}$ is a noise vector. $U_{1}, U_{2}, U_{3}$, and $U_{4}$ as the inputs of the quad-rotor are adjusted by four motor rotor speeds. Control input is selected as

$$
\mathbf{u}=\left[\begin{array}{c}
-\frac{1}{m} U_{1} U_{x} \\
-\frac{1}{m} U_{1} U_{y} \\
\frac{1}{m}\left(\cos \phi \cos \theta U_{1}\right) \\
\frac{l}{J_{x}} U_{2} \\
\frac{l}{J_{y}} U_{3} \\
\frac{1}{J_{z}} U_{4}
\end{array}\right]
$$

3.1. Sliding Mode Controller of Quad-Rotor UAV. Let the desired state of quad-rotor be $X_{d}=\left[x_{d}, y_{d}, z_{d}, \phi_{d}, \theta_{d}, \psi_{d}\right]^{T}$. Then the state tracking error of the system $\mathbf{e}_{X}^{(\alpha)}$ can be described as follows:

$$
\mathbf{e}_{X}^{(\alpha)}=X_{d}^{(\alpha)}-X^{(\alpha)}
$$


where $\mathbf{e}_{X}^{(\alpha)} \in R^{6}$ and $\alpha$ is the factorial number. Sliding surface equation is given by

$$
\begin{array}{ll} 
& \mathbf{s}_{X}=\mathbf{e}_{X}^{(\alpha)}+\sum_{i=1}^{\alpha-1} \lambda_{X(\alpha-i)} \frac{d^{i} \mathbf{e}_{X}}{d t^{i}}+\lambda_{X \alpha} \mathbf{e}_{X} \\
\text { s.t. } & \mathbf{s}_{X} \dot{\mathbf{s}}_{X}<-\beta_{X}\left|\mathbf{s}_{X}\right|<0 .
\end{array}
$$

Because $\lambda_{X(\alpha-i)}$ conforms to Hurwitz stable polynomial, $\beta_{X}>0$, the sliding mode motion is asymptotically stable. The input includes two parts can be written as

$$
\begin{aligned}
U_{\mathrm{eg}} & =\mathbf{g}(X)^{-1}\left[-\mathbf{f}(X)-\Delta \mathbf{f}(X)+\frac{d^{(\alpha+1)} X_{d}}{d t^{(\alpha+1)}}\right. \\
& \left.-\sum_{i=1}^{\alpha-1} \lambda_{X(\alpha-i)} \frac{d^{i+1} \mathbf{e}_{X}}{d t^{i+1}}-\lambda_{X \alpha} \frac{d \mathbf{e}_{X}}{d t}\right], \\
U_{\mathrm{sw}} & =-\mathbf{g}(X)^{-1} \mathbf{k} \operatorname{sign}\left(s_{X}\right),
\end{aligned}
$$

where $U_{\text {eg }}$ is the equivalent control part and $U_{s w}$ is the switch part for the purpose of suppressing systematic disturbances. Let $\mathbf{k}=\mathbf{d}+\boldsymbol{\eta}$, where $\boldsymbol{\eta}$ is greater than zero.

It can be written as $\mathbf{u}=U_{\mathrm{eg}}+U_{\mathrm{sw}}$. Similarly, $U_{2}, U_{3}$, and $U_{4}$ can be obtained by

$$
\begin{aligned}
U_{2} & =\frac{J_{x}}{l}\left[-\frac{J_{y}-J_{z}}{J_{x}} q r-\frac{J_{r z}}{J_{x}} \Omega^{\prime} q-\frac{k_{D x} p}{J_{x}}-d_{\phi}\right. \\
& \left.+\lambda_{\phi 1} \frac{d^{2} \phi_{d}}{d t^{2}}-\lambda_{\phi 2} \frac{d e_{\phi}}{d t}\right]-\frac{J_{x}}{l} k_{\phi} \operatorname{sign}\left(s_{\phi}\right), \\
U_{3} & =\frac{J_{y}}{l}\left[-\frac{J_{z}-J_{x}}{J_{y}} p r+\frac{J_{r z}}{J_{y}} \Omega^{\prime} p-\frac{k_{D y} q}{J_{x}}-d_{\theta}\right. \\
& \left.+\lambda_{\theta 1} \frac{d^{2} \theta_{d}}{d t^{2}-\lambda_{\theta 2}} \frac{d e_{\theta}}{d t}\right]-\frac{J_{y}}{l} k_{\theta} \operatorname{sign}\left(s_{\theta}\right) \\
U_{4} & =J_{z}\left[-\frac{J_{x}-J_{y}}{J_{z}} p q-\frac{k_{D z} r}{J_{x}}-d_{\psi}+\lambda_{\psi 1} \frac{d^{2} \psi_{d}}{d t^{2}}\right. \\
& \left.-\lambda_{\psi 2} \frac{d e_{\psi}}{d t}\right]-J_{z} k_{\psi} \operatorname{sign}\left(s_{\psi}\right),
\end{aligned}
$$

where $k_{\phi}>0, k_{\theta}>0$, and $k_{\psi}>0$. This system will tend toward origin point along the sliding surface $s$ by the action of the sliding mode control inputs.

The sliding mode controller receives the difference between desired position $\left(x_{d}, y_{d}, z_{d}\right)$ and the real-time position $\left(x_{t}, y_{t}, z_{t}\right)$ of quad-rotor UAV. After calculating the control input $U_{1}$, the two virtual control inputs $U_{x}, U_{y}$ can be obtained. Virtual controllers are joined into the attitude algorithm module and then the desired attitude angles $\phi_{d}$, $\theta_{d}$ are calculated. Further, the desired attitude angles $\phi_{d}, \theta_{d}$, $\psi_{d}$ together with the returned attitude angles $\phi, \theta, \psi$ are sent to the attitude control system, where they can be promoted to control the quad-rotor UAV flight attitude. Similar to the sliding mode control design for attitude system, the position errors are defined as follows:

$$
\begin{aligned}
& e_{x}=x_{d}-x_{t}, \\
& e_{y}=y_{d}-y_{t}, \\
& e_{z}=z_{d}-z_{t} .
\end{aligned}
$$

Derivation process is similar to (18) and (19) and the sliding mode surfaces $\left(s_{x}, s_{y}, s_{z}\right)$ of $(x, y, z)$ can be got by using the position error and their derivatives. Finally, control inputs $U_{1}, U_{x}$, and $U_{y}$ are designed, respectively, as follows:

$$
\begin{aligned}
U_{1} & =\frac{m}{(\cos \phi \cos \theta)}\left[g-k_{R 3} \dot{z}+\lambda_{z 1} \frac{d^{2} z_{d}}{d t^{2}}-\lambda_{z 2} \frac{d e_{z}}{d t}\right. \\
& \left.-d_{z}\right]-\frac{m}{(\cos \phi \cos \theta)} k_{z} \operatorname{sign}\left(s_{z}\right), \\
U_{x} & =\frac{m}{U_{1}}\left[-k_{R 1} \dot{x}+\lambda_{x 1} \frac{d^{2} x_{d}}{d t^{2}}-\lambda_{x 2} \frac{d e_{x}}{d t}-d_{x}\right]-\frac{m}{U_{1}} \\
& \cdot k_{x} \operatorname{sign}\left(s_{x}\right), \\
U_{y} & =\frac{m}{U_{1}}\left[-k_{R 2} \dot{y}+\lambda_{y 1} \frac{d^{2} y_{d}}{d t^{2}}-\lambda_{y 2} \frac{d e_{y}}{d t}-d_{y}\right]-\frac{m}{U_{1}} \\
& \cdot k_{y} \operatorname{sign}\left(s_{y}\right) .
\end{aligned}
$$

According to (22), through the virtual control inputs $U_{x}$, $U_{y}$ to get $\ddot{x}, \ddot{y}$, thus the desired pitch angle and roll angle are calculated. The inverse equations are given by

$$
\begin{aligned}
& \phi_{d}=\arcsin \left[-\frac{(\sin \psi m \ddot{x}-\cos \psi m \ddot{y})}{T}\right], \\
& \theta_{d}=\arcsin \left[-\frac{(\cos \psi m \ddot{x}+\sin \psi m \ddot{y})}{T \cos \phi}\right] .
\end{aligned}
$$

3.2. Offline SVM Modeling of Quad-Rotor UAV with Unknown Parameters. Some nonlinear terms and parameters of a quad-rotor UAV are usually unknown, which may disturb the precision of the model. Aiming at the disadvantages of physical model, this paper adopts the offline SVM to establish the quad-rotor UAV model. For a given training sample data set $\left\{\left(\mathbf{x}_{i}, \mathbf{f}_{i}\right) \mid \mathbf{x}_{i} \in R^{n}, y_{i} \in R, i=1,2, \ldots, L\right\}, L$ is the number of sample data and SVM prediction function equation can be written as

$$
\mathbf{f}(\mathbf{x})=w^{T} K\left(\mathbf{x}_{i}, \mathbf{x}\right)+b
$$

where $K\left(\mathbf{x}_{i}, \mathbf{x}\right)=\exp \left(-\left\|\mathbf{x}_{i}-\mathbf{x}\right\|^{2} / 2 \sigma^{2}\right)$ is a RBF kernel function, $b$ is the intercept number, and $\sigma$ is the width parameter of 
the RBF kernel function. According to the structural risk minimization principle

$$
\begin{array}{ll}
\min & J(w)=\frac{1}{2}\langle w, w\rangle+C \sum_{t i=1}^{L}\left(\xi_{i}+\xi_{i}^{*}\right), \\
\text { s.t. } & f\left(\mathbf{x}_{i}\right)-w^{T} K\left(\mathbf{x}_{i}, \mathbf{x}\right)-b \leq \varepsilon+\xi_{i} \\
& w^{T} K\left(\mathbf{x}_{i}, \mathbf{x}\right)+b-f\left(\mathbf{x}_{i}\right) \leq \varepsilon+\xi_{i}^{*} \\
& i=1,2, \ldots, L
\end{array}
$$

where $C$ is the penalty parameter, $\varepsilon$ is the insensitive loss function, and $\xi_{i}, \xi_{i}^{*}$ are the relaxation factors. Lagrange function is established as follows to solve the above optimization problem:

$$
\begin{aligned}
& L\left(w, b, \varepsilon, \xi_{i}, \xi_{i}^{*}, \lambda_{i}, \lambda_{i}^{*}\right) \\
& =J(w)-\sum_{i=1}^{L} \lambda_{i}\left[f\left(\mathbf{x}_{i}\right)-w^{T} K\left(\mathbf{x}_{i}, \mathbf{x}\right)-b-\varepsilon-\xi_{i}\right] \\
& \quad-\sum_{i=1}^{L} \lambda_{i}^{*}\left[w^{T} K\left(\mathbf{x}_{i}, \mathbf{x}\right)+b-\varepsilon-\xi_{i}^{*}-f\left(\mathbf{x}_{i}\right)\right],
\end{aligned}
$$

where $\lambda_{i}, \lambda_{i}^{*}$ are Lagrange multipliers. According to the KKT (Karush-Kuhn-Tucker) condition, eliminating $\varepsilon, \xi_{i}, \xi_{i}^{*}$, and $w$, the following SVM nonlinear function can be obtained by

$$
\mathbf{f}(\mathbf{x})=\sum_{i=1}^{m}\left(\lambda_{i}-\lambda_{i}^{*}\right) K\left(\mathbf{x}_{i}, \mathbf{x}\right)+b .
$$

3.3. Online Adaptive SVM Error Compensations of Uncertainties and Noises. It is relatively hard to obtain a completely precise estimation of model utilizing the offline SVM once regression. Other uncertain factors such as modeling error and uncertainties in the flight process will also influence the stability of the UAV during the flight. Therefore, this paper combines the online adaptive SVM with offline SVM for compensation of modeling errors which are caused by the uncertainties and noises during the flight process. Define $D(\mathbf{x})=\mathbf{f}(X)-\widehat{\mathbf{f}}\left(\mathbf{x} \mid w^{\prime}\right)+\Delta \mathbf{f}+\mathbf{d}$, where $\widehat{\mathbf{f}}\left(\mathbf{x} \mid w^{\prime}\right)$ represents the estimated value of $\mathbf{f}(X)$ and $w^{\prime}=\lambda_{i}-\lambda_{i}^{*}$. According to the characteristic value $\mathbf{x}_{i}$ in the sample data, the predicted value $\widehat{D}_{i}$ with respect to the $D_{i}$ is given by

$$
\begin{aligned}
\widehat{D}_{i}\left(\mathbf{x} \mid w_{i}^{\prime \prime}\right) & =\sum_{i=1}^{L}\left(\lambda_{D i}-\lambda_{D i}^{*}\right) K\left(\mathbf{x}_{i}, \mathbf{x}\right)+b^{\prime} \\
& =\sum_{i=1}^{L} w_{i}^{\prime \prime} K\left(\mathbf{x}_{i}, \mathbf{x}\right)+b^{\prime}
\end{aligned}
$$

where $\lambda_{D i}, \lambda_{D i}^{*}$ are the computed Lagrange multipliers of $\widehat{D}_{i}\left(\mathbf{x} \mid w_{i}^{\prime \prime}\right)$ and $w_{i}^{\prime \prime}=\lambda_{D i}-\lambda_{D i}^{*}$. Then the boundary function of the $i$ th sample can be defined as

$$
\begin{aligned}
h\left(\mathbf{x}_{i} \mid w_{j}^{\prime \prime}\right) & =D\left(\mathbf{x} \mid w_{j}^{\prime \prime}\right)-\widehat{D}\left(\mathbf{x} \mid w_{j}^{\prime \prime}\right) \\
& =\sum_{j=1}^{m} w_{j}^{\prime \prime} K\left(\mathbf{x}_{i}, \mathbf{x}_{j}\right)+b-\widehat{D}\left(\mathbf{x} \mid w_{j}^{\prime \prime}\right) .
\end{aligned}
$$

According to the KKT condition, a congruent relationship between $h\left(\mathbf{x}_{i} \mid w_{j}^{\prime \prime}\right)$ and $w_{j}^{\prime \prime}$ can be obtained as follows [26]:

$$
\begin{aligned}
& h\left(\mathbf{x}_{i} \mid w_{j}^{\prime \prime}\right) \geq+\varepsilon \quad w_{j}^{\prime \prime}=-C, \\
& h\left(\mathbf{x}_{i} \mid w_{j}^{\prime \prime}\right)=+\varepsilon \quad w_{j}^{\prime \prime} \in[-C, 0], \\
& h\left(\mathbf{x}_{i} \mid w_{j}^{\prime \prime}\right) \in[-\varepsilon,+\varepsilon] \quad w_{j}^{\prime \prime}=-C, \\
& h\left(\mathbf{x}_{i} \mid w_{j}^{\prime \prime}\right)=-\varepsilon \quad w_{j}^{\prime \prime} \in[0,+C], \\
& h\left(\mathbf{x}_{i} \mid w_{j}^{\prime \prime}\right) \leq-\varepsilon \quad w_{j}^{\prime \prime}=+C .
\end{aligned}
$$

According to the size of $D_{i}(\mathbf{x})$, it can be divided into the following three kinds of circumstances: support vector error set $E=\left\{i|| w_{j}^{\prime \prime} \mid=C\right\}$, support vector set $S=\left\{i|0<| w_{j}^{\prime \prime} \mid<\right.$ $C\}$, and retain sample set $R=\left\{i \mid w_{j}^{\prime \prime}=0\right\}$.

If a new sample $\mathbf{x}_{l}$ is added here, parameters $\Delta w^{\prime \prime}, \Delta b^{\prime}$ can be obtained by computing. Because each sample satisfies KKT condition, the change of available boundary function value is given by

$$
\begin{aligned}
\Delta h\left(\mathbf{x}_{i} \mid w_{j}^{\prime \prime}\right)= & K\left(\mathbf{x}_{i}, \mathbf{x}_{l}\right) \Delta w_{l}^{\prime \prime} \\
& +\sum_{j=1}^{L} K\left(\mathbf{x}_{i}, \mathbf{x}_{j}\right) \Delta w_{j}^{\prime \prime}+\Delta b^{\prime} .
\end{aligned}
$$

And because the sum of $\Delta w^{\prime \prime}$ is always equal to zero,

$$
\sum_{j \in S} w_{j}^{\prime \prime}+w_{l}^{\prime \prime}=0
$$

According to (31) and (32), it can be found in the support vector set $S$ as follows:

$$
\begin{aligned}
\sum_{j \in S} K\left(\mathbf{x}_{i}, \mathbf{x}_{j}\right) \Delta w_{j}^{\prime \prime}+\Delta b & =-K\left(\mathbf{x}_{i}, \mathbf{x}_{l}\right) \Delta w_{l}^{\prime \prime}, \\
\sum_{j \in S} \Delta w_{j}^{\prime \prime} & =-\Delta w_{l}^{\prime \prime} .
\end{aligned}
$$




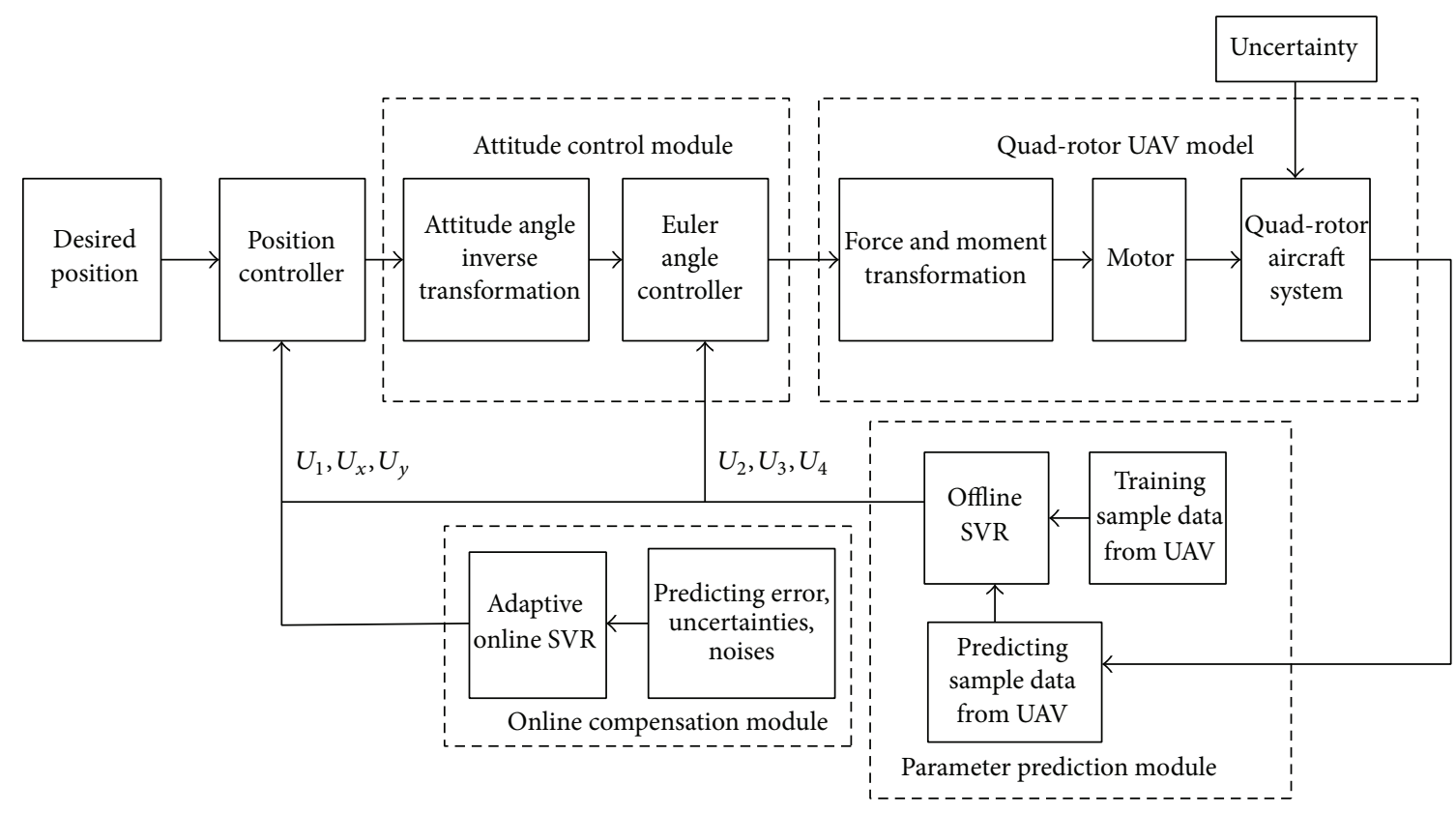

Figure 3: Position control system structure for quad-rotor UAV.

The support vector set is defined as $S=\left\{s_{1}, s_{2}, \ldots, s_{L}\right\}$. According to (33), the following matrix can be given by

$$
\begin{aligned}
\left(\begin{array}{c}
\Delta b \\
\Delta w_{s_{1}}^{\prime \prime} \\
\vdots \\
\Delta w_{s_{m}}^{\prime \prime}
\end{array}\right) & \\
= & -\left(\begin{array}{cccc}
0 & 1 & \cdots & 1 \\
1 & K\left(\mathbf{x}_{s_{1}}, \mathbf{x}_{s_{1}}\right) & \cdots & K\left(\mathbf{x}_{s_{1}}, \mathbf{x}_{s_{m}}\right) \\
\vdots & \vdots & \ddots & \vdots \\
1 & K\left(\mathbf{x}_{s_{m}}, \mathbf{x}_{s_{1}}\right) & \cdots & K\left(\mathbf{x}_{s_{m}}, \mathbf{x}_{s_{m}}\right)
\end{array}\right) \\
& \left(\begin{array}{c}
1 \\
K\left(\mathbf{x}_{s_{1}}, \mathbf{x}_{s_{l}}\right) \\
\vdots \\
K\left(\mathbf{x}_{s_{m}}, \mathbf{x}_{s_{l}}\right)
\end{array}\right) \Delta w_{l}^{\prime \prime}=\eta \Delta w_{l}^{\prime \prime},
\end{aligned}
$$

where

$$
\begin{aligned}
\eta= & -\left(\begin{array}{cccc}
0 & 1 & \cdots & 1 \\
1 & K\left(\mathbf{x}_{s_{1}}, \mathbf{x}_{s_{1}}\right) & \cdots & K\left(\mathbf{x}_{s_{1}}, \mathbf{x}_{s_{m}}\right) \\
\vdots & \vdots & \ddots & \vdots \\
1 & K\left(\mathbf{x}_{s_{m}}, \mathbf{x}_{s_{1}}\right) & \cdots & K\left(\mathbf{x}_{s_{m}}, \mathbf{x}_{s_{m}}\right)
\end{array}\right)^{-1} \\
& \cdot\left(\begin{array}{c}
1 \\
K\left(\mathbf{x}_{s_{1}}, \mathbf{x}_{s_{l}}\right) \\
\vdots \\
K\left(\mathbf{x}_{s_{m}}, \mathbf{x}_{s_{l}}\right)
\end{array}\right) .
\end{aligned}
$$

It can change $w_{j}^{\prime \prime}$ but cannot change the $h$ of the support vectors set. However, it will alter $h$ for the support vector error set and retain sample set. Assume that $N=E \cup R=$ $\left\{n_{1}, n_{2}, \ldots, n_{N}\right\}$; the changes of $h$ can be got from (31), (35), and (36)

$$
\begin{gathered}
\left(\begin{array}{c}
\Delta h\left(\mathbf{x}_{n_{1}} \mid w_{s_{l}}^{\prime \prime}\right) \\
\Delta h\left(\mathbf{x}_{n_{2}} \mid w_{s_{l}}^{\prime \prime}\right) \\
\vdots \\
\Delta h\left(\mathbf{x}_{n_{N}} \mid w_{s_{l}}^{\prime \prime}\right)
\end{array}\right) \\
=\left(\begin{array}{c}
\Delta K\left(\mathbf{x}_{n_{1}}, \mathbf{x}_{s_{l}}\right) \\
\Delta K\left(\mathbf{x}_{n_{2}}, \mathbf{x}_{s_{l}}\right) \\
\vdots \\
\Delta K\left(\mathbf{x}_{n_{N}}, \mathbf{x}_{s_{l}}\right)
\end{array}\right) \Delta w_{l}^{\prime \prime}
\end{gathered}
$$

$$
+\left(\begin{array}{cccc}
0 & K\left(\mathbf{x}_{n_{1}}, \mathbf{x}_{s_{1}}\right) & \cdots & K\left(\mathbf{x}_{n_{1}}, \mathbf{x}_{s_{m}}\right) \\
1 & K\left(\mathbf{x}_{n_{2}}, \mathbf{x}_{s_{1}}\right) & \cdots & K\left(\mathbf{x}_{n_{2}}, \mathbf{x}_{s_{m}}\right) \\
\vdots & \vdots & \ddots & \vdots \\
1 & K\left(\mathbf{x}_{n_{N}}, \mathbf{x}_{s_{1}}\right) & \cdots & K\left(\mathbf{x}_{n_{N}}, \mathbf{x}_{s_{m}}\right)
\end{array}\right) \eta \Delta w_{l}^{\prime \prime} .
$$

So every set can be renewed and adjusted to achieve the objective that online SVM learns the noises and the uncertainties of the model through the above equations. Position control system structure diagram of the corresponding sliding mode controller is shown in Figure 3. 

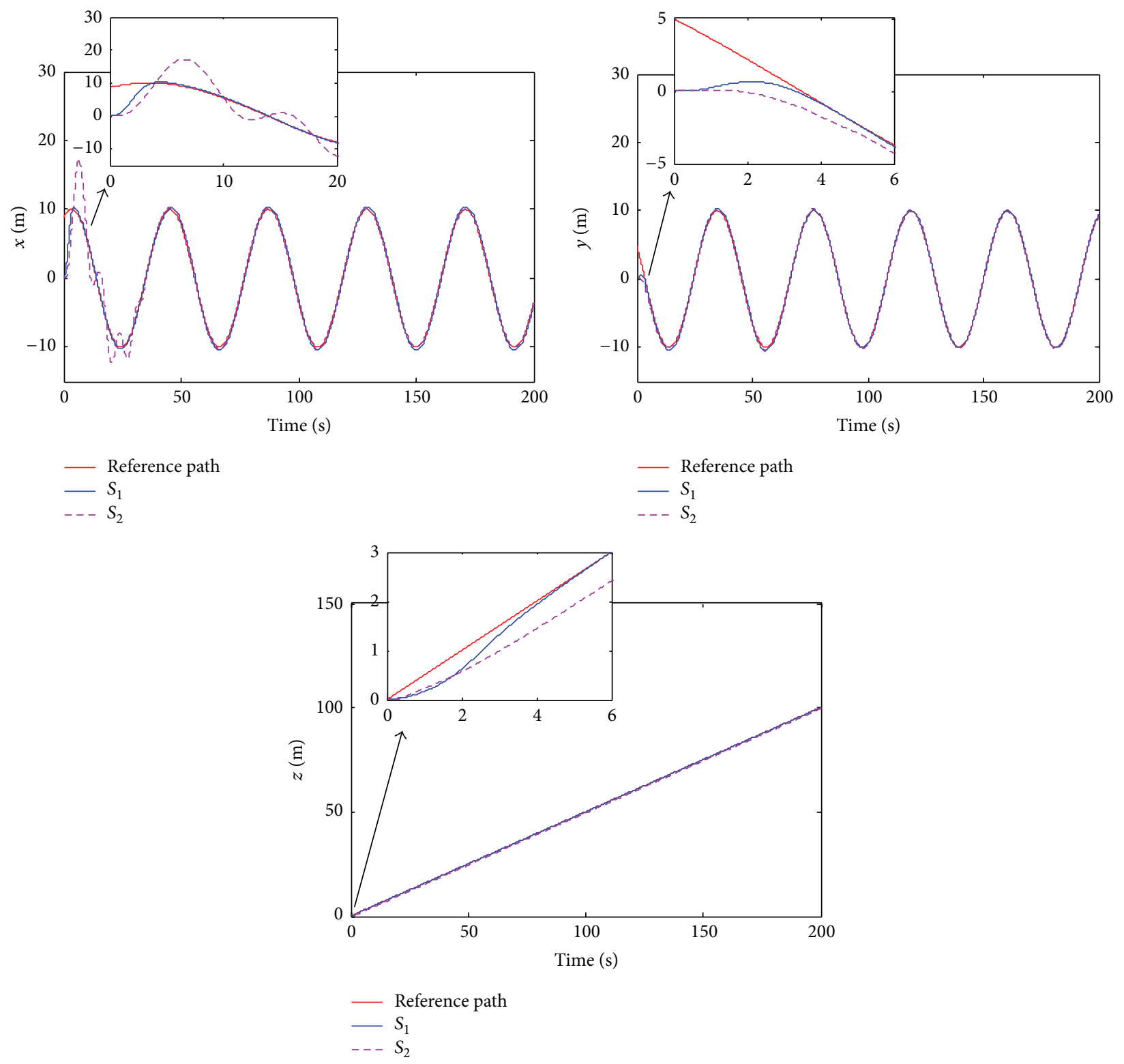

FIGURE 4: UAV flight path tracking results of the positions $(x, y, z)$.

\section{Stability Analysis of Online Adaptive Error Compensation SVM}

According to (19), (27), and (28), the sliding mode controller is redesigned as

$$
\begin{aligned}
\mathbf{u} & =\mathbf{g}(X)^{-1}\left[-\widehat{\mathbf{f}}\left(X \mid w^{\prime}\right)-\widehat{D}\left(X \mid w^{\prime \prime}\right)+\frac{d^{(\alpha+1)} X_{d}}{d t^{(\alpha+1)}}\right. \\
& \left.-\sum_{i=1}^{\alpha-1} \lambda_{X(\alpha-i)} \frac{d^{i+1} \mathbf{e}_{X}}{d t^{i+1}}-\lambda_{X \alpha} \frac{d \mathbf{e}_{X}}{d t}-\mathbf{k} \operatorname{sign}\left(s_{X}\right)\right] .
\end{aligned}
$$

Then

$$
\begin{aligned}
\widehat{\mathbf{f}}\left(X \mid w^{\prime}\right)+\widehat{D}\left(X \mid w^{\prime \prime}\right) \\
=\sum_{i=1}^{s v} w^{\prime} K\left(\mathbf{x}_{i}, \mathbf{x}_{j}\right)+\sum_{i=1}^{s v^{\prime}} w^{\prime \prime} K\left(\mathbf{x}_{i}, \mathbf{x}_{j}\right),
\end{aligned}
$$

where $w^{\prime}$ and $w^{\prime \prime}$ are support vector weights of offline SVM and online SVM, respectively.

Assumption 1. Outputs $\widehat{\mathbf{f}}\left(X \mid w^{\prime}\right)$ and $\widehat{D}\left(X \mid w^{\prime \prime}\right)$ of offline SVM and online SVM are continuous and bounded. 

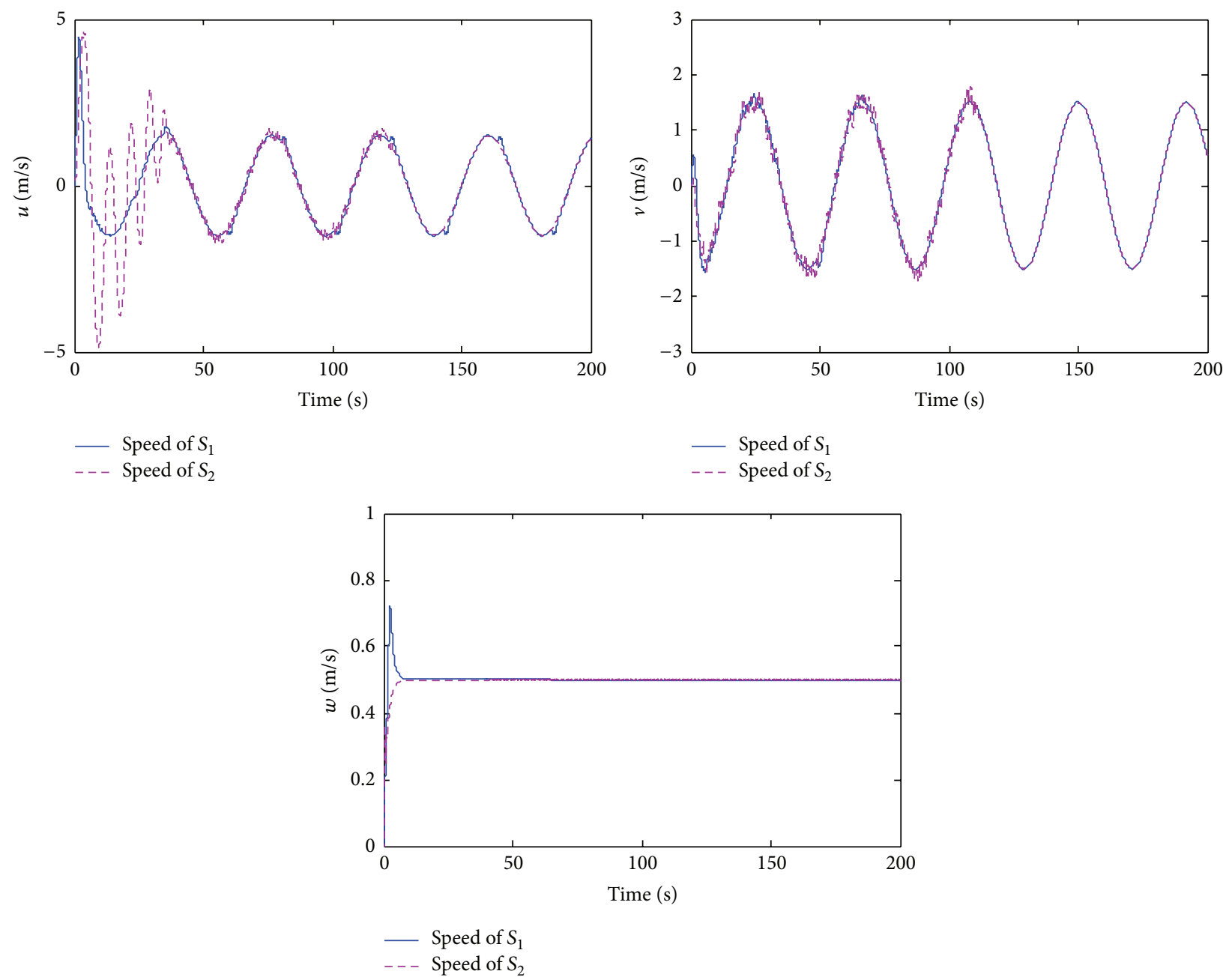

FIGURE 5: UAV flight path tracking results of the speeds $(u, v, w)$.

Assumption 2. Outputs $\widehat{\mathbf{f}}\left(X \mid w^{\prime}\right)$ and $\widehat{D}\left(X \mid w^{\prime \prime}\right)$ can approximate $F(X)=\mathbf{f}(X)+\Delta \mathbf{f}+\mathbf{d}$, if there is a very small positive number $\varepsilon_{0}$ that satisfies $\max \| F-\widehat{\mathbf{f}}\left(X \mid w^{\prime}\right)-\widehat{D}(X \mid$ $\left.w^{\prime \prime}\right) \| \leq \varepsilon_{0}$.

If $\mathbf{f}^{*}\left(X \mid w^{*}\right)=\max \left\|\widehat{\mathbf{f}}\left(X \mid w^{\prime}\right)+\widehat{D}\left(X \mid w^{\prime \prime}\right)\right\|=$ $\sum_{i=1}^{s v} w^{*} K\left(\mathbf{x}_{i}, \mathbf{x}_{j}\right)$, where $w^{*}=\arg \min [\sup \mid F(X)-\widehat{\mathbf{f}}(X \mid$ $\left.\left.w^{\prime}\right)-\widehat{D}\left(X \mid w^{\prime \prime}\right) \mid\right]$ is the optimal weight vector, then $F(X)=$ $\mathbf{f}^{*}\left(X \mid w^{*}\right)+\zeta$ that satisfies $\zeta \leq \varepsilon_{0}$; let $\widetilde{w}=w^{*}-w^{\prime}-w^{\prime \prime}$.

Theorem 3. For the nonlinear system (14), using the modified sliding mode control law (38), the sliding mode control system is asymptotically stable if the adaptive adjusting law is $\dot{\bar{w}}=$ $-\gamma K\left(\mathbf{x}_{i}, \mathbf{x}_{j}\right) s^{T}$, where $\gamma>0$ is the gain parameter of adaptive rate.

Proof. The following Lyapunov function is considered:

$$
V=\frac{1}{2} s_{X}^{T} s_{X}+\frac{1}{2 \gamma} \widetilde{w}^{T} \widetilde{w} .
$$

The derivative of (18) is given by

$$
\begin{aligned}
\dot{s}_{X}= & \mathbf{e}_{X}^{(\alpha+1)}+\sum_{i=1}^{\alpha-1} \lambda_{X(\alpha-i)} \frac{d^{i+1} \mathbf{e}_{X}}{d t^{i+1}}+\lambda_{X \alpha} \frac{d \mathbf{e}_{X}}{d t} \\
= & \frac{d^{(\alpha+1)} X}{d t^{(\alpha+1)}}-\frac{d^{(\alpha+1)} X_{d}}{d t^{(\alpha+1)}} \\
& +\sum_{i=1}^{\alpha-1} \lambda_{X(\alpha-i)} \frac{d^{i+1} \mathbf{e}_{X}}{d t^{i+1}}+\lambda_{X \alpha} \frac{d \mathbf{e}_{X}}{d t} .
\end{aligned}
$$

Using (14) and letting $d=0$, then

$$
\begin{aligned}
\dot{s}_{X}= & F(X)+g(X) \mathbf{u}-\frac{d^{(\alpha+1)} X_{d}}{d t^{(\alpha+1)}} \\
& +\sum_{i=1}^{\alpha-1} \lambda_{X(\alpha-i)} \frac{d^{i+1} \mathbf{e}_{X}}{d t^{i+1}}+\lambda_{X \alpha} \frac{d \mathbf{e}_{X}}{d t} .
\end{aligned}
$$



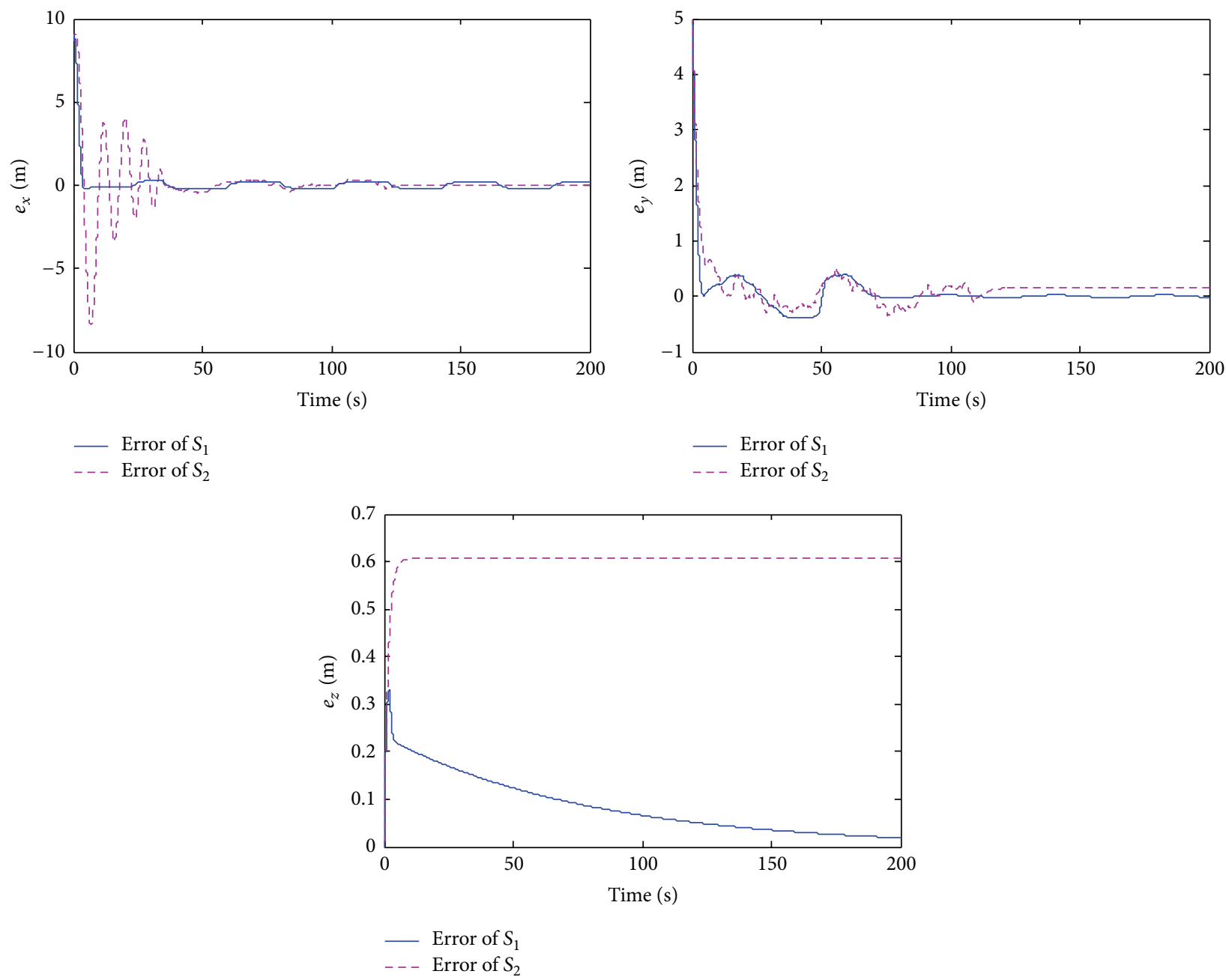

FIGURE 6: The tracking errors of the positions $(x, y, z)$.

By substituting (38) into (42), (42) becomes

$$
\begin{aligned}
\dot{s}_{X}= & F(X)-\widehat{\mathbf{f}}\left(X \mid w^{\prime}\right)-\widehat{D}\left(X \mid w^{\prime \prime}\right)-\mathbf{k} \operatorname{sign}\left(s_{X}\right) \\
= & \mathbf{f}^{*}\left(X \mid w^{*}\right)-\widehat{\mathbf{f}}\left(X \mid w^{\prime}\right)-\widehat{D}\left(X \mid w^{\prime \prime}\right) \\
& -\mathbf{k} \operatorname{sign}\left(s_{X}\right)+\zeta \\
= & \widetilde{w} K\left(\mathbf{x}_{i}, \mathbf{x}_{j}\right)-\mathbf{k} \operatorname{sign}\left(s_{X}\right)+\zeta .
\end{aligned}
$$

Using (43) and taking derivative of (40) yield

$$
\begin{aligned}
\dot{V}= & s_{X}{ }^{T} \dot{s}_{X}+\frac{1}{\gamma} \widetilde{w}^{T} \dot{\tilde{w}} \\
= & s_{X}{ }^{T}\left(\zeta-\mathbf{k} \operatorname{sign}\left(s_{X}\right)\right) \\
& +\frac{1}{\gamma} \operatorname{tr}\left(\dot{\tilde{w}} \widetilde{w}^{T}+\gamma K\left(\mathbf{x}_{i}, \mathbf{x}_{j}\right) s_{X}{ }^{T} \widetilde{w}^{T}\right) .
\end{aligned}
$$

Because $\dot{\widetilde{w}}_{\phi}=\dot{w}_{\phi}^{*}-\dot{w}_{\phi}^{\prime}-\dot{w}_{\phi}^{\prime \prime}$ and $\dot{w}_{\phi}^{*}=\dot{w}_{\phi}=0$, the adaptive adjusting law is selected as

$$
\dot{\tilde{w}}=-\dot{w}^{\prime}=-\gamma K\left(\mathbf{x}_{i}, \mathbf{x}_{j}\right) s_{X}^{T} .
$$

Then (44) can be written as

$$
\dot{V}=s_{X}^{T}\left(\zeta-\mathbf{k} \operatorname{sign}\left(s_{X}\right)\right) \text {. }
$$

The boundary value of $\zeta$ is $\zeta_{0}$. It can be known that $\dot{V} \leq 0$ if $k \geq \zeta_{0}$. Not only the asymptotic stability of the system and the boundedness of $\widetilde{w}$ are ensured, but also the system outputs tend to the desired target $X_{d}$.

\section{Simulation Results}

To verify the effectiveness of the control method, Matlab/Simulink simulation platform is introduced. This platform is responsible for simulating the position tracking control of the quad-rotor UAV system with multiple flight aim points under the condition of high frequency noises 


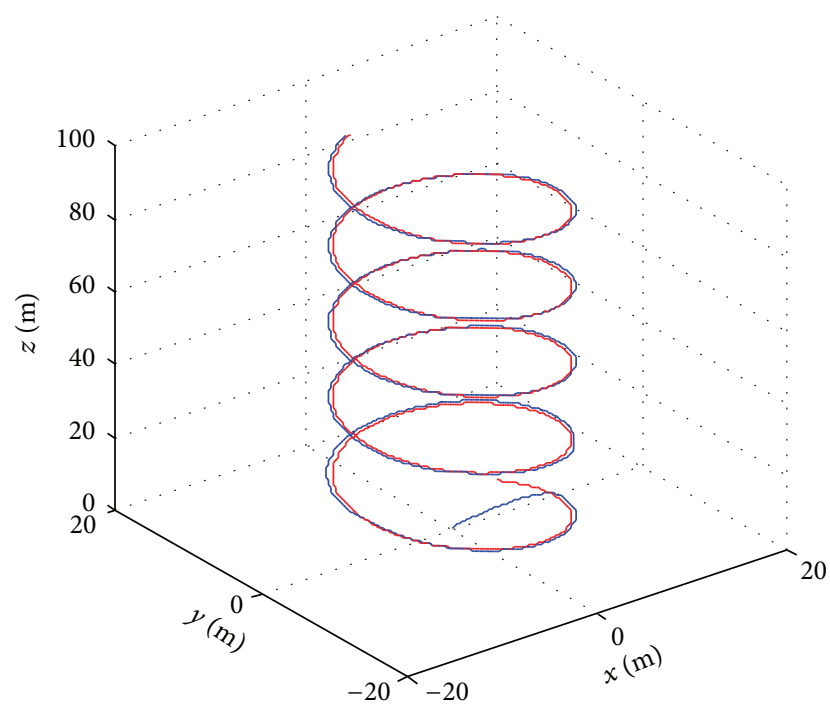

Reference path
$S_{1}$

FIGURE 7: The 3D path tracking of $S_{1}$.

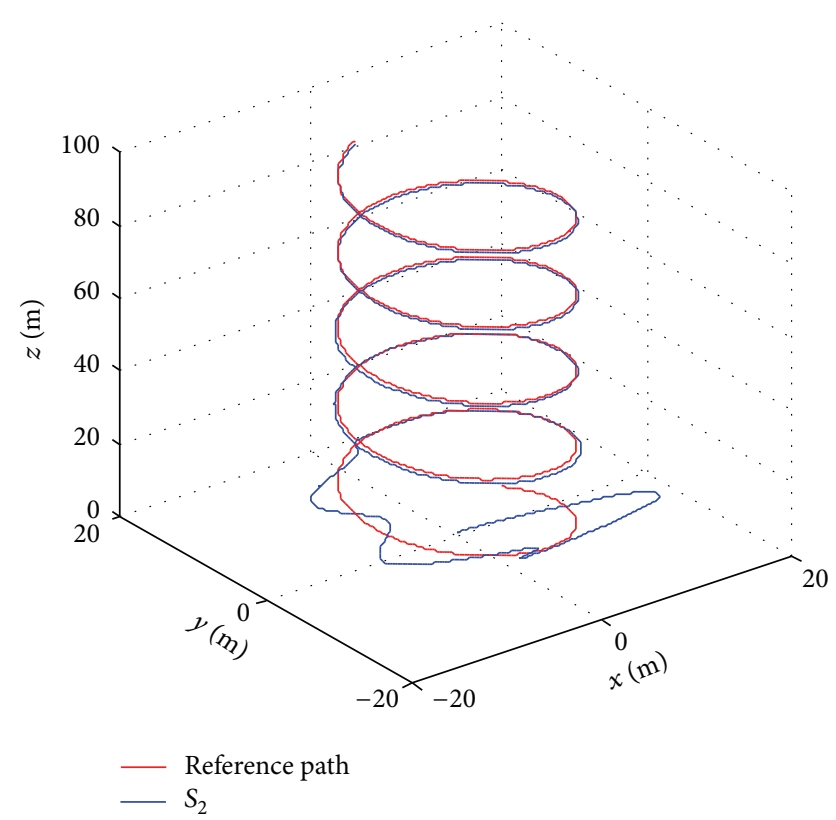

Figure 8: The 3D path tracking of $S_{2}$.

and uncertainties. At the same time, the attitude controller output is recorded. Control goal is to make the position track reference instructions.

First, part parameters of quad-rotor Unmanned Aerial Vehicle are given in Table 1. The sliding mode controller (19) is established by using the offline SVM to predict the model when there are some unknown parameters in the UAV system. Training sample data include the speed of each motor and the aircraft attitude. Sampling period is $0.01 \mathrm{~s}$ and 2000 points are taken as training sample data.
TABLE 1: Quad-rotor parameters in simulation.

\begin{tabular}{lc}
\hline Parameter & Value \\
\hline$m$ & $0.468 \mathrm{~kg}$ \\
$g$ & $9.81 \mathrm{~m} / \mathrm{s}^{2}$ \\
$l$ & $0.225 \mathrm{~m}$ \\
$k_{b}$ & $2.98 \times 10^{-6} \mathrm{~N} \cdot \mathrm{s} / \mathrm{rad}^{2}$ \\
$k_{d}$ & $1.14 \times 10^{-7} \mathrm{~N} \cdot \mathrm{m} \cdot \mathrm{s} / \mathrm{rad}^{2}$ \\
$J_{x}$ & $4.856 \times 10^{-3} \mathrm{~kg} \cdot \mathrm{m}^{2}$ \\
$J_{y}$ & $4.856 \times 10^{-3} \mathrm{~kg} \cdot \mathrm{m}^{2}$ \\
$J_{z}$ & $8.801 \times 10^{-3} \mathrm{~kg} \cdot \mathrm{m}^{2}$ \\
$J_{r x}$ & $6.15 \times 10^{-5} \mathrm{~kg} \cdot \mathrm{m}^{2}$ \\
$J_{r y}$ & $6.15 \times 10^{-5} \mathrm{~kg} \cdot \mathrm{m}^{2}$ \\
\hline
\end{tabular}

At the same time, the sliding mode controller is reconstructed by joining online adaptive SVM compensation. There are 30 online SVM training samples and replaced by the new data real-timely. Adaptive parameter $\gamma$ of online SVM is taken as 1.5 .

The start point of flight is $(0,0,0) \mathrm{m}$ and UAV tracks the desired paths $x_{d}=10 \sin (0.15 t+\pi / 3) \mathrm{m}, y_{d}=10 \sin (0.15 t+$ $\pi * 5 / 6) \mathrm{m}$, and $z_{d}=0.5 t \mathrm{~m}$. The simulation time is $200 \mathrm{~s}$. Both controllers are joined to the same UAV system under the conditions of existing noise which is equal to $0.4 * \sin (50 * X)$ and $20 \%$ uncertainties including mass, inertia, and aerodynamic coefficients. UAV flight path with online adaptive error compensation SVM-based sliding mode control was labeled as $S_{1}$, and the flight path with only offline SVM-based sliding mode control was labeled as $S_{2}$. The flight path tracking results of two conditions are shown in Figure 4.

And the speeds $u, v$, and $w$ of the quad-rotor are shown in Figure 5.

It can be seen that the UAV with the sliding mode control based on online adaptive SVM compensation can track the path more rapidly and accurately even if there are some noises and uncertainties. It also can be seen that the UAV which joins online adaptive SVM compensation has the good performance from the tracking error. Changes of the position errors $e_{x}, e_{y}$, and $e_{z}$ are shown in Figure 6.

The $3 \mathrm{D}$ path tracking with two different controllers is shown in Figures 7 and 8.

In order to test the step signal response of UAV with online adaptive error compensation SVM-based sliding mode control, let UAV fly from the initial position $(0,0,0) \mathrm{m}$ through points $(3,3,8) \mathrm{m},(12,7,8) \mathrm{m}$, and $(13,14,8) \mathrm{m}$ in turn. The simulation time is $60 \mathrm{~s}$ and position simulation results are shown as in Figure 9.

Figure 9 shows that the UAV with online adaptive SVM compensation reaches the three target points at $9 \mathrm{~s}, 28 \mathrm{~s}$, and $47 \mathrm{~s}$. And the path without online adaptive SVM compensation is more slow. Three-dimensional path tracking diagrams are shown in Figure 10, Figure 10(a) is the expected route, and Figures 10(b), 10(c), and 10(d) are the actual paths of UAV in the different time points.

As shown in Figure 9, although there are uncertainties and noises, it still can achieve the satisfactory tracking. Good results have two reasons. First, offline SVM and online 

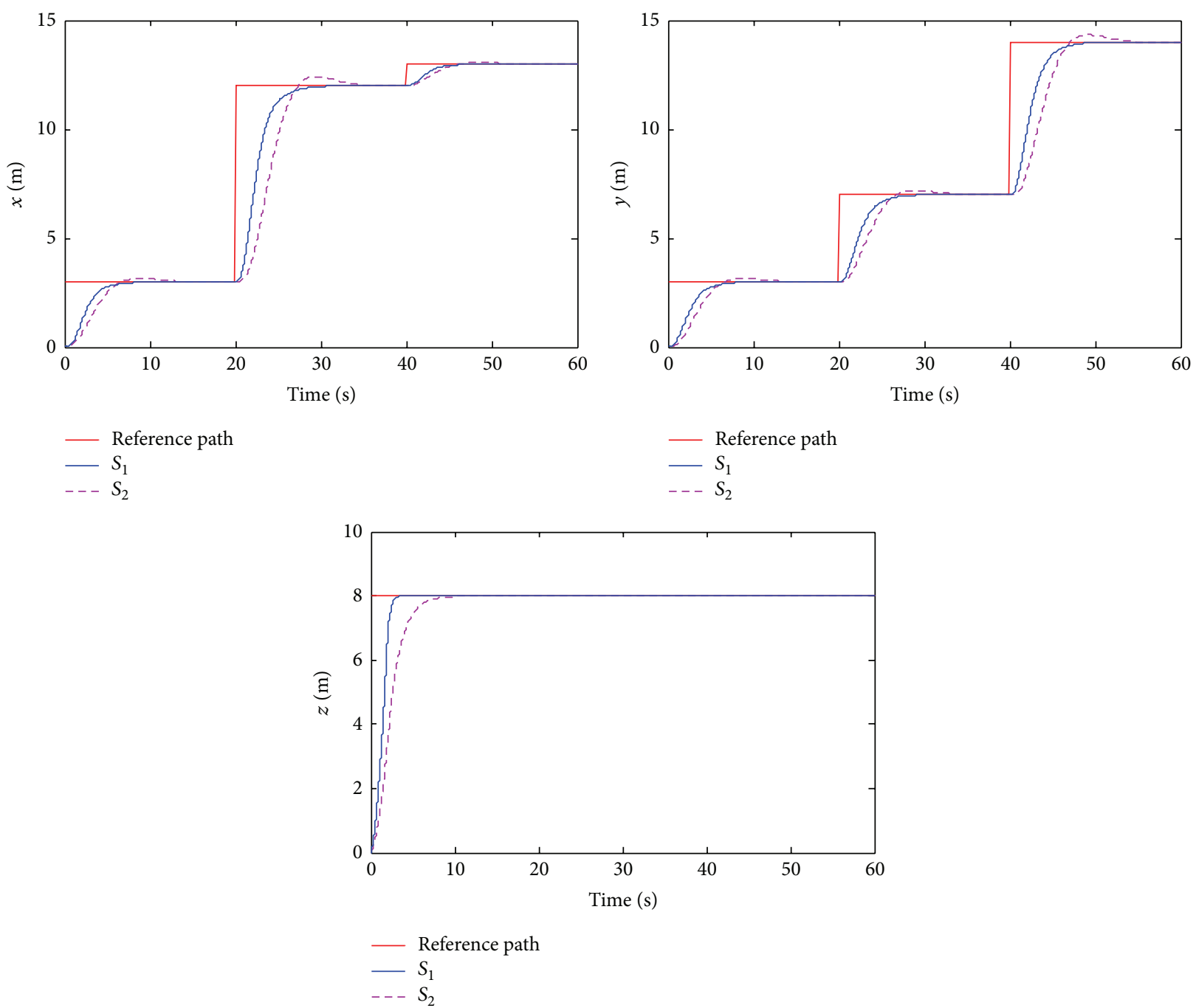

FIGURE 9: UAV path tracking of the positions $(x, y, z)$.

adaptive SVM used together can establish the model rapidly and the errors will be compensated real-timely even if there are noises and uncertainties. Second, as the adaptive online SVM real-time adjustment method, it will not lead to a decline in tracking performance even if the predictions have deviation or drift.

It can be seen from the results of simulation, whatever, modeling errors, parameter uncertainties or noises do not affect the tracking accuracy of the controller. And the robustness of the controller can be proved.

\section{Conclusion}

In this paper, the control problem of quad-rotor UAV system is discussed during tracking the procedure by using the sliding mode controller. To ensure the stabilization and the navigation of UAV with unknown parameters, a sliding mode controller with online adaptive error compensation SVM is presented. Sliding mode controller is established through analyzing quad-rotor dynamics model in which the unknown parameters are estimated by offline SVM. But offline SVM cannot guarantee the precision of model in the process of flight because modeling errors and external disturbances always exist. So the online adaptive SVM method is added for the controller to compensate noises and uncertainties. Control law is adjusted in real-time by introducing new training sample data to online adaptive SVM in the control process and thereby it guarantees the stability and the robustness of flight.

Two experiments about above control scheme are performed. In the first experiment, it can be shown that the control performance with only offline SVM modeling is worse than the control performance with both offline SVM and online adaptive SVM. And the tracking speed is much slower. In the second experiment, the step signal response of UAV is tested with online adaptive error compensation SVMbased sliding mode control. The simulation results also verify its superiority and the better results are obtained in this case. 


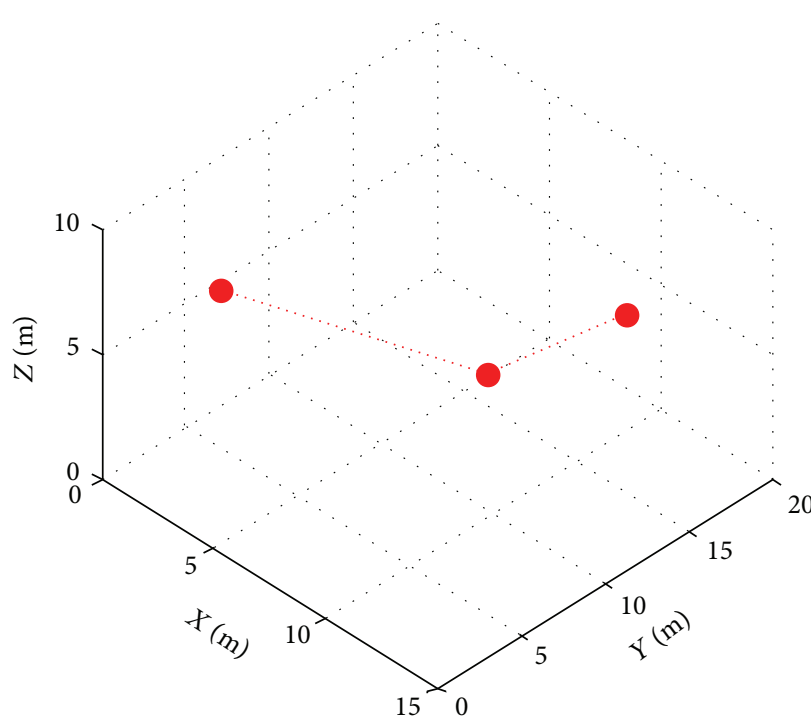

Reference path

(a) $t=0 \mathrm{~s}$

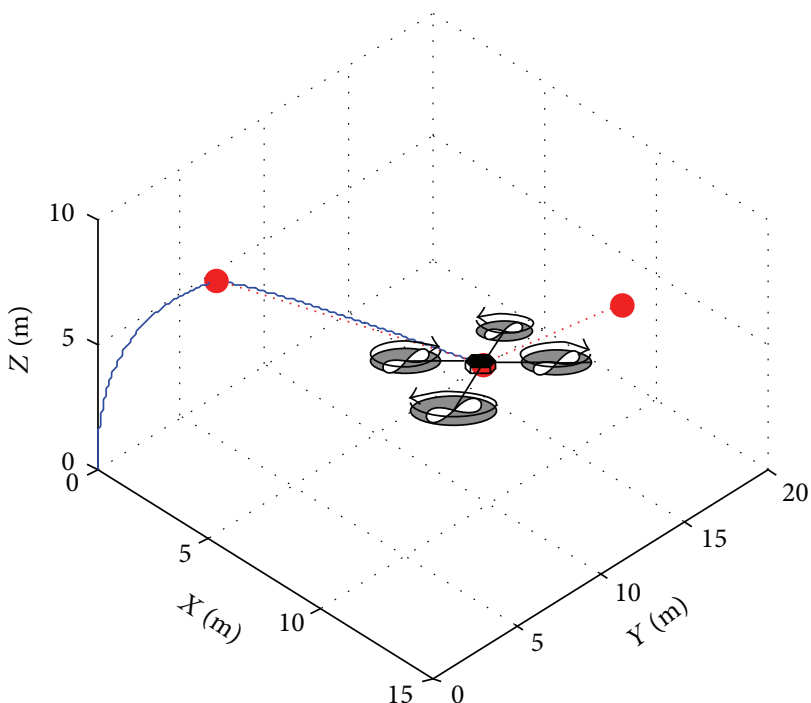

Reference path

— Actual path

(c) $t=28 \mathrm{~s}$

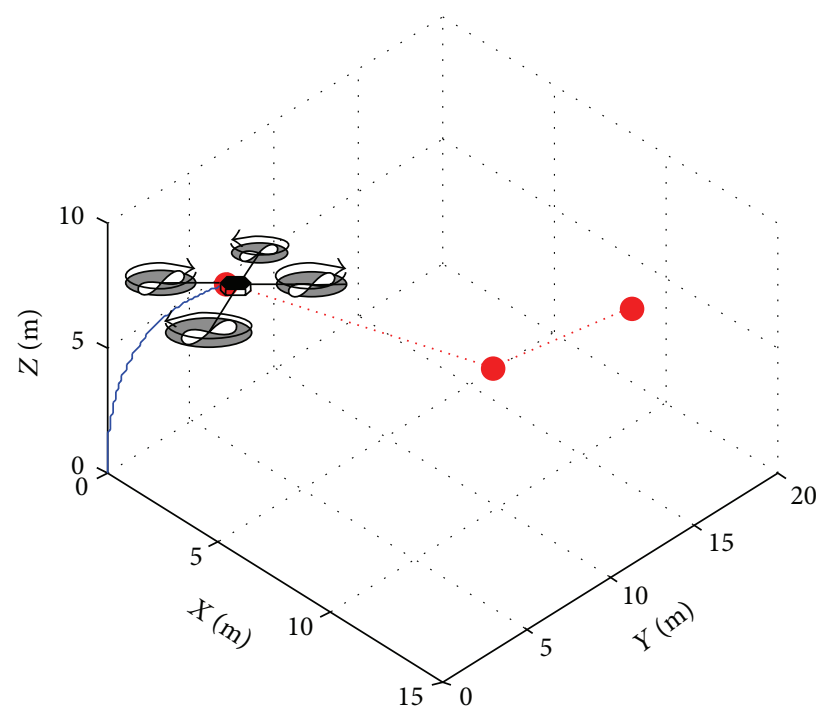

Reference path

- Actual path

(b) $t=9 \mathrm{~s}$

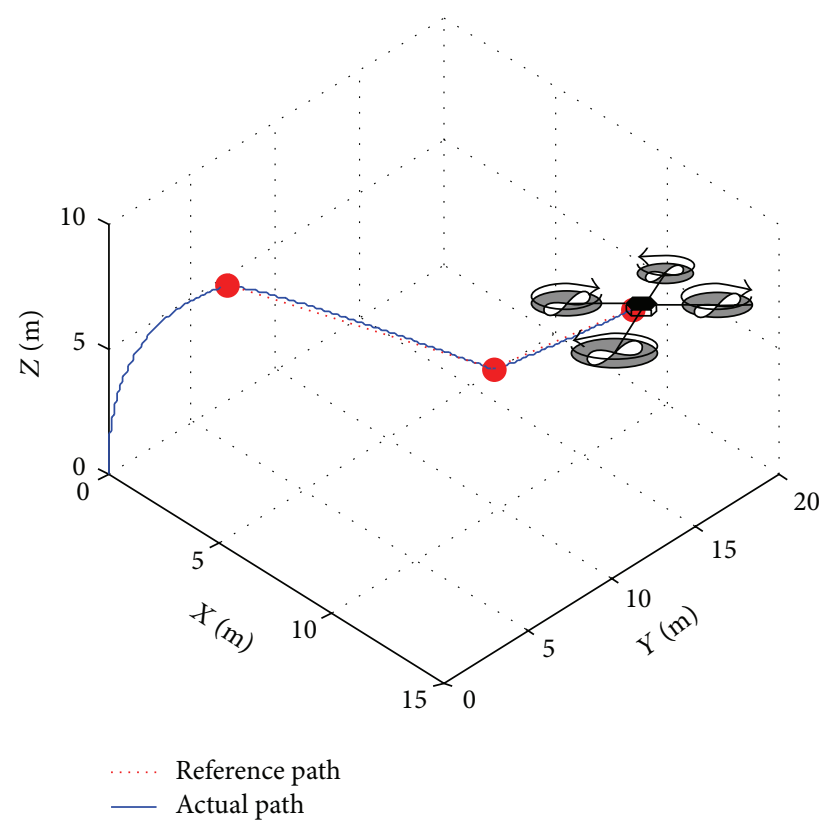

(d) $t=47 \mathrm{~s}$

FIgURE 10: Three-dimensional UAV path tracking.

The simulation results demonstrate that the proposed method effectively improves the path tracking performance. They also prove the good performance of online adaptive error compensation SVM-based sliding mode control in a quad-rotor UAV system with noises and uncertainties.

\section{Conflict of Interests}

The authors declare that there is no conflict of interests regarding the publication of this paper.

\section{Acknowledgment}

This work was supported by JiangSu Scientific Support Program of China (Grant no. BE2014712).

\section{References}

[1] C. Ha, Z. Zuo, F. B. Choi, and D. Lee, "Passivity-based adaptive backstepping control of quadrotor-type UAVs," Robotics and Autonomous Systems, vol. 62, no. 9, pp. 1305-1315, 2014. 
[2] A. Modirrousta and M. Khodabandeh, "A novel nonlinear hybrid controller design for an uncertain quadrotor with disturbances," Aerospace Science and Technology, vol. 45, pp. 294-308, 2015.

[3] A. Nagaty, S. Saeedi, C. Thibault, M. Seto, and H. Li, "Control and navigation framework for quadrotor helicopters," Journal of Intelligent \& Robotic Systems, vol. 70, no. 1-4, pp. 1-12, 2013.

[4] G. Nikolakopoulos and K. Alexis, "Switching networked attitude control of an unmanned quadrotor," International Journal of Control, Automation and Systems, vol. 11, no. 2, pp. 389-397, 2013.

[5] K. Alexis, G. Nikolakopoulos, and A. Tzes, "Experimental constrained optimal attitude control of a quadrotor subject to wind disturbances," International Journal of Control, Automation and Systems, vol. 12, no. 6, pp. 1289-1302, 2014.

[6] V. Roldão, R. Cunha, D. Cabecinhas, C. Silvestre, and P. Oliveira, "A leader-following trajectory generator with application to quadrotor formation flight," Robotics and Autonomous Systems, vol. 62, no. 10, pp. 1597-1609, 2014.

[7] S. A. Belokon', Y. N. Zolotukhin, A. S. Mal'tsev, A. A. Nesterov, M. N. Filippov, and A. P. Yan, "Control of flight parameters of a quadrotor vehicle moving over a given trajectory," Optoelectronics, Instrumentation and Data Processing, vol. 48, no. 5, pp. 454-461, 2012.

[8] Y. Yali, S. Feng, and W. Yuanxi, "Controller design of quadrotor aerial robot," Physics Procedia, vol. 33, pp. 1254-1260, 2012.

[9] H. A. F. Almurib, P. T. Nathan, and T. N. Kumar, "Control and path planning of quadrotor aerial vehicles for search and rescue," in Proceedings of the SICE Annual Conference (SICE '11), Tokyo, Japan, September 2011.

[10] K. Alexis, G. Nikolakopoulos, A. Tzes, and L. Dritsas, "Coordination of helicopter UAVs for aerial forest-fire surveillance," in Applications of Intelligent Control to Engineering Systems, $\mathrm{K}$. P. Valavanis, Ed., vol. 39 of Intelligent Systems, Control, and Automation: Science and Engineering, pp. 169-193, 2009.

[11] C.-C. Li, G.-S. Zhang, T.-J. Lei, and A.-D. Gong, "Quick imageprocessing method of UAV without control points data in earthquake disaster area," Transactions of Nonferrous Metals Society of China, vol. 21, no. 3, pp. s523-s528, 2011.

[12] F. Fraundorfer, L. Heng, D. Honegger et al., "Vision-based autonomous mapping and exploration using a quadrotor MAV," in Proceedings of the 25th IEEE/RSJ International Conference on Robotics and Intelligent Systems (IROS '12), pp. 4557-4564, IEEE, Vilamoura, Portugal, October 2012.

[13] P. J. Zarco-Tejada, R. Diaz-Varela, V. Angileri, and P. Loudjani, "Tree height quantification using very high resolution imagery acquired from an unmanned aerial vehicle (UAV) and automatic 3D photo-reconstruction methods," European Journal of Agronomy, vol. 55, pp. 89-99, 2014.

[14] F. Nex and F. Remondino, "UAV for 3D mapping applications: a review," Applied Geomatics, vol. 6, no. 1, pp. 1-15, 2014.

[15] N. Metni and T. Hamel, "A UAV for bridge inspection: visual servoing control law with orientation limits," Automation in Construction, vol. 17, no. 1, pp. 3-10, 2007.

[16] J. Baluja, M. P. Diago, P. Balda et al., "Assessment of vineyard water status variability by thermal and multispectral imagery using an unmanned aerial vehicle (UAV)," Irrigation Science, vol. 30 , no. 6 , pp. 511-522, 2012.

[17] F. Chen, B. Jiang, and G. Tao, "An intelligent self-repairing control for nonlinear MIMO systems via adaptive sliding mode control technology," Journal of the Franklin Institute, vol. 351, no. 1, pp. 399-411, 2014.
[18] R. Ashok and Y. Shtessel, "Control of fuel cell-based electric power system using adaptive sliding mode control and observation techniques," Journal of the Franklin Institute, vol. 352, no. 11, pp. 4911-4934, 2015.

[19] Y. Singh and M. Santhakumar, "Inverse dynamics and robust sliding mode control of a planar parallel (2-PRP and 1-PPR) robot augmented with a nonlinear disturbance observer," Mechanism and Machine Theory, vol. 92, pp. 29-50, 2015.

[20] V. Azhmyakov, "On the set-valued approach to optimal control of sliding mode processes," Journal of the Franklin Institute, vol. 349, no. 4, pp. 1323-1336, 2012.

[21] A. Al-Khazraji, N. Essounbouli, A. Hamzaoui, F. Nollet, and J. Zaytoon, "Type-2 fuzzy sliding mode control without reaching phase for nonlinear system," Engineering Applications of Artificial Intelligence, vol. 24, no. 1, pp. 23-38, 2011.

[22] T.-C. Lin, M.-C. Chen, and M. Roopaei, "Synchronization of uncertain chaotic systems based on adaptive type-2 fuzzy sliding mode control," Engineering Applications of Artificial Intelligence, vol. 24, no. 1, pp. 39-49, 2011.

[23] C.-F. Hsu, "Adaptive neural complementary sliding-mode control via functional-linked wavelet neural network," Engineering Applications of Artificial Intelligence, vol. 26, no. 4, pp. 1221-1229, 2013.

[24] F. G. Rossomando, C. Soria, and R. Carelli, "Adaptive neural sliding mode compensator for a class of nonlinear systems with unmodeled uncertainties," Engineering Applications of Artificial Intelligence, vol. 26, no. 10, pp. 2251-2259, 2013.

[25] Y. G. Kao, C. H. Wang, H. R. Karimi, and W. Li, " $H_{\infty}$ sliding mode control for uncertain neutral-type stochastic systems with Markovian jumping parameters," Information Sciences, vol. 314, pp. 200-211, 2015.

[26] M. Castro-Neto, Y.-S. Jeong, M.-K. Jeong, and L. D. Han, "Online-SVR for short-term traffic flow prediction under typical and atypical traffic conditions," Expert Systems with Applications, vol. 36, no. 3, pp. 6164-6173, 2009. 


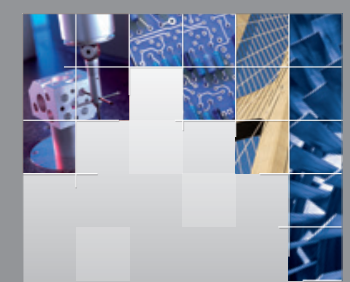

\section{Enfincering}
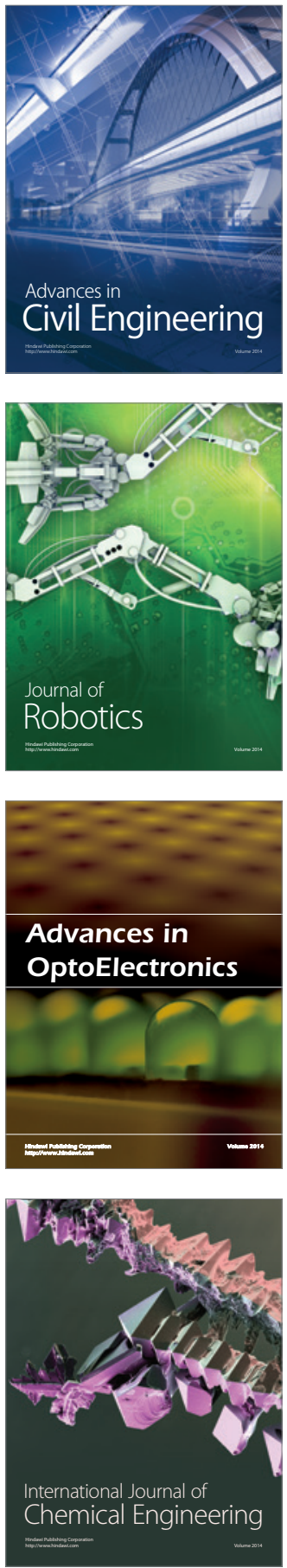

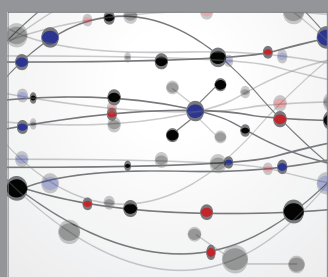

The Scientific World Journal

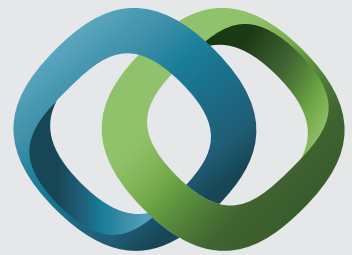

\section{Hindawi}

Submit your manuscripts at

http://www.hindawi.com
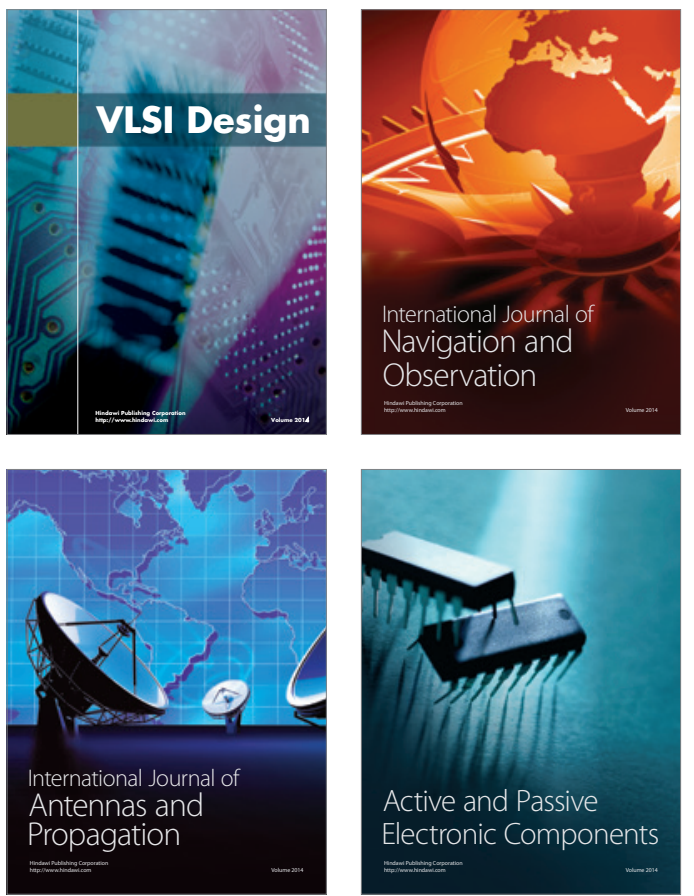
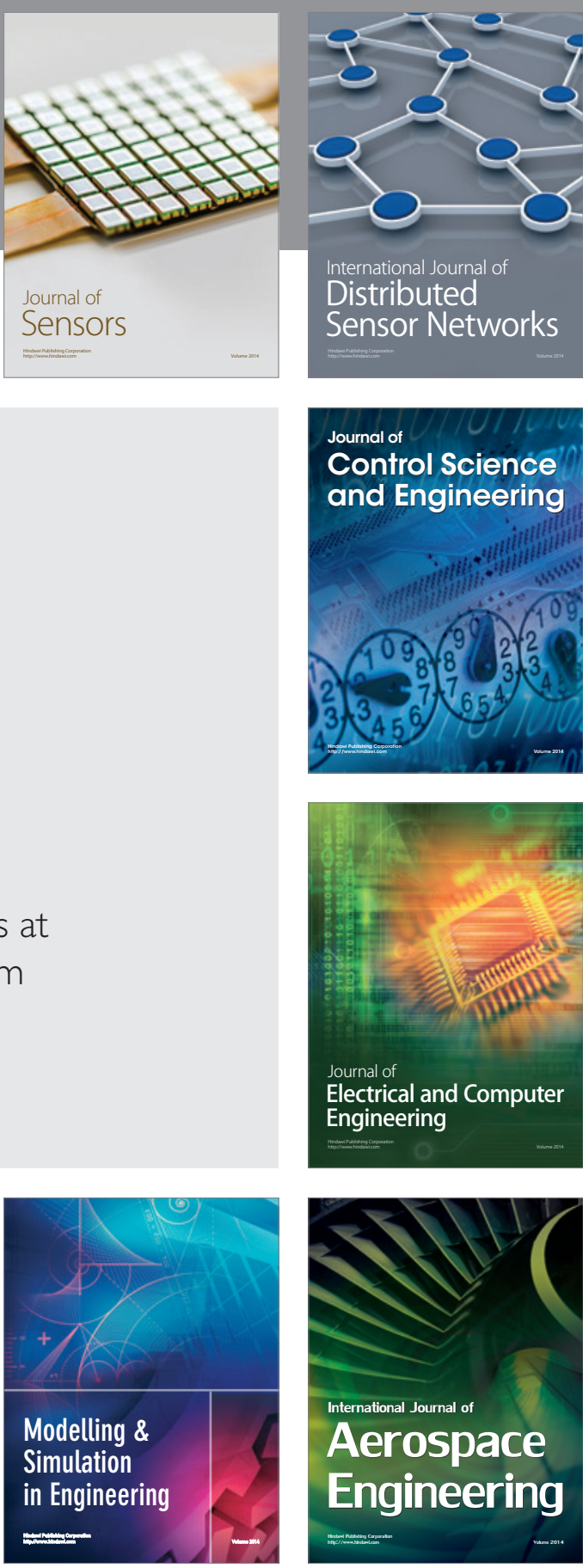

International Journal of

Distributed

Sensor Networks

Journal of

Control Science

and Engineering
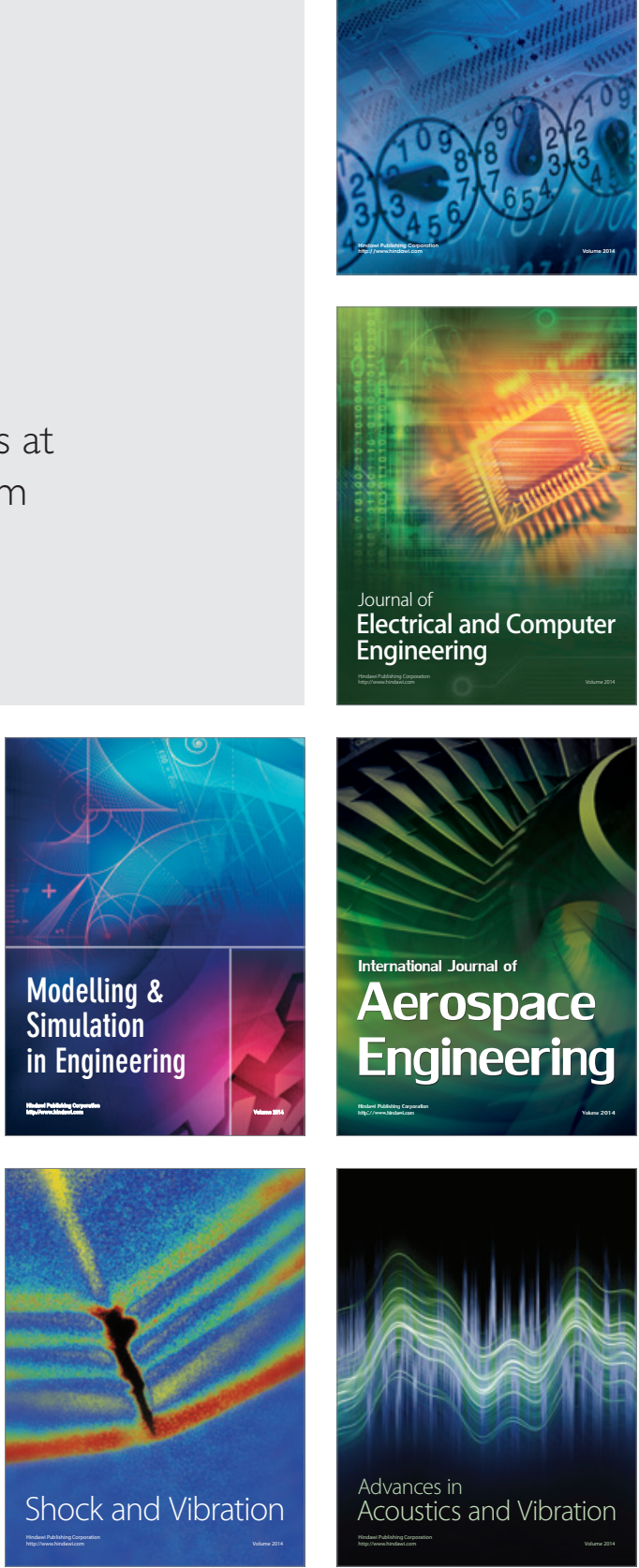